\title{
TRIAGEM VIRTUAL PARA A SELEÇÃO DE INIBIDORES DUAIS FRENTE À ACETILCOLINESTERASE E BUTIRILCOLINESTERASE HUMANA
}

\author{
Ana Mercia Silva Mascarenhas'; Franco Henrique Andrade Leite²; Moysés Fagundes de \\ Araújo Neto $^{3}$; Raquel Bianca Marchesine de Almeida ${ }^{4}$; Mariana Borges Botura ${ }^{5}$. \\ 1. Bolsista FAPESB, Graduanda em Farmácia, Universidade Estadual de Feira de Santana, e-mail: anamercia.sm@gmail.com \\ 2. Orientador, Departamento de Saúde, Universidade Estadual de Feira de Santana, e-mail: fhpharm@gmail.com \\ 3. Laboratório de Modelagem Molecular, Universidade Estadual de Feira de Santana, e-mail: moysesfagundes@ gmail.com \\ 4. Laboratório de Toxicologia, Universidade Estadual de Feira de Santana, e-mail: raquelbma87@gmail.com \\ 5. Laboratório de Toxicologia, Universidade Estadual de Feira de Santana, e-mail: mbbotura@uefs.br
}

PALAVRAS-CHAVE: Acetilcolinesterase; Butirilcolinesterase; Triagem virtual.

\section{INTRODUÇÃO}

Acetilcolinesterase (AChE) e Butirilcolinesterase (BuChE) estão envolvidas na transmissão colinérgica,e sua deficiência é considerada uma das principais hipóteses da patogênese da Doença de Alzheimer (DA) (FALCO et al., 2016). Por esse motivo, o tratamento da DA é baseado principalmente na monoterapia com fármacos anticolinérgicos com seletividade para AChE (ex.: donezepila e galantamina) e/ou ambos os receptores (ex.: rivastigmina). Contudo, a monoterapia é ineficaz devido ao efeito compensatório entre a AChE e BuChE (NAJAFI et al., 2017), além disso os fármacos atuais possuem baixa eficácia e alto perfil de efeitos adversos e portanto a identificação de novos inibidoresduaistorna-se uma alternativa promissora para o tratamento de pacientes com DA. Uma forma de alcançar esse objetivo é através da integraçãode estratégias químico-medicinais capazes de identificar requisitos estéreo-eletrônicos essenciais para a inibição de ambos os alvos biológicos (ex. modelos farmacofóricos) e que forneçam informações de afinidade e modo de ligação no sítio ativo (ex. acoplamento molecular)(RODRIGUES et al., 2012). Diante do exposto, a triagem virtualpor modelos farmacofóricos e acoplamento molecular foi utilizada para priorizar potenciais inibidores duais frente a $\mathrm{AChE}$ e $\mathrm{BuChE}$, presentes no banco de moléculas da SigmaAldrich $^{\circledR}$ disponível na plataforma ZINC ${ }^{15}$.

\section{METODOLOGIA}

Seleção dos inibidores: 18 inibidores da acetilcolinesterase (E.C 3.1.1.7; AChE) e butirilcolinesterase humana (E.C 3.1.1.8; BuChE), com $\mathrm{Ki}<50 \mu \mathrm{M}$ e índice de seletividade BuChE/AChE entre 0,6 e 1,4, foram coletados da literatura (SUN et al,. 2014) e posteriormente divididos em treino $(n=7)$ e teste $(n=11)$ para a geração e avaliação dos modelos farmacofóricos, respectivamente. As estruturas 2D foram obtidas no programa Marvin ${ }^{\circledR}$ Sketch 15.4.20 (CHEMAXON, 2015) e convertidas para o formato 3D na plataforma SYBYL®-X 2.0 (TRIPOS, 2010). Cargas parciais foram calculadas pelo método Gaisteiger-Huckel ele aplicado o protocolo de minimização (CG = 0,001 Kcal/mol; Campo de força Tripos) (TRIPOS, 2010).

Geração dos modelos farmacofóricos: Os modelos farmacofóricos foram gerados atravésdo alinhamento flexível dos confôrmeros entre si pelo de algoritmo genético implementado no módulo GALAHAD padrão (Population size $=X$; Max generation=Y). Os operadores genéticos foram mantidos nos seus valores.

Avaliação dos modelos farmacofóricos: Os modelos farmacofóricos com ENERGY < 100 $\mathrm{Kcal} / \mathrm{mol}$ e PARETO diferente de zeroforam avaliados quanto a habilidade de diferenciar ativos de falsos positivos (decoys) construídos com o auxílio do servidor DUD-E numa proporção de 1:50 (MYSINGERet al., 2012). Após o alinhamento com o banco contendo todas as moléculas (ativos + falso positivo) foi construída a curva ROC (do inglês, Receiver Operating Characteristic) e calculado a AUC (do inglês, área under the curve) de cada modelo. Os modelos farmacofóricos com AUC > 0,70 foram selecionados e posteriormente submetidos ao cálculo do valor da discriminação aprimorada de Boltzmann da curva ROC (do inglês, Boltzmann-Enhanced Discrimination of ROC - BEDROC) através do servidor online ROCKER (LÄTTI; NIINIVEHMAS; PENTIKÄINEN, 2016). Por fim, os modelos remanescentesforam testados 
quanto à capacidade de relacionar o encaixe dos compostos no farmacóforo (QFIT) com sua respectiva atividade biológica $(\mathrm{pKi}=\log 1 / \mathrm{Ki})$. O modelo com a melhor relação entre potência e QFITfoi utilizado para filtrar o banco de moléculas do ZINC disponível para aquisição na plataforma Sigma-Aldrich ${ }^{\circledR}$.

Acoplamento molecular: As estruturas 3D da AChE (PDB: 4M0E) e BuChE (PDB: 4BDS) foram preparadas com auxílio do módulo Biopolymer. Asmoléculas de água não estruturais e demais artefatos de cristalização foram excluídos. Foram adicionados átomos de hidrogênio e cargas Gaisteiger-Huckel. O estado de protonação foi avaliado através do servidor PropKa 3.0 (SONDERGAARD et al., 2011). As etapas de busca conformacional e avaliação das poses foram testadas no AutoDockVina 1.1.2 (TROTT \& OLSON, 2010). Os parâmetros de busca foram avaliados quanto a capacidade de identificar a pose cristalográfica através do redocking com a melhor pose (RMSD < $2 \AA$ ) e da capacidade de reconhecimento de ativos em relação a falsos positivos através do cálculo da área sob a curva ROC (AUC >0,7). As moléculas remanescentes da triagem por modelo farmacofórico foram submetidas ao acoplamento molecularfrente às estruturas 3D de AChE e BuChE. As moléculas com mediana menor que -9,0e -8,6 kcal/mol para AChE e BuChE respectivamente foram analisadas quanto a Eficiência de ligação. Moléculas com eficiência de ligação maior que $-0,4 \mathrm{kcal} / \mathrm{mol}$ (HOPKINS et al., 2014) foram excluídas. Logo após, a moléculas com características comuns a $\mathrm{AChE}$ e BuChE foram selecionadas para as etapas seguintes. A aquisição das moléculas levou em consideração a disponibilidade comercial e custo (< $100,0 \mathrm{US} \$ / 10 \mathrm{mg}$ ). Por fim, as moléculas adquiridas foram avaliadas quanto aos filtros moleculares de Lipinski (1997) e Veber et al.(2002).

\section{RESULTADOS E DISCUSSÃO}

A busca por fármacos duais frente a DA é crucial para o controle da doença haja vista a ineficácia do tratamento em monoterapia. A aplicação de métodos computacionais têm possibilitado uma taxa de enriquecimento superior aos métodos randômicos para a identificação de moléculas bioativas. Dentre essas técnicas, o modelo farmacofórico merece destaque, pois é capaz de identificar requisitos estéreo-eletrônicos essenciais para inibição de alvos específicos e, portanto, são uteis na priorização de moléculas candidatas a ensaios biológicos. Por esse motivo, 10 modelos farmacofóricos foram gerados e após a avaliação dos parâmetros estatísticos internos (Energy < 100 $\mathrm{kcal} / \mathrm{mol}$; Pareto $=0$ ) todos os modelos foram testados quando a taxa de acerto para a identificação de inibidores verdadeiros (Figura 1).

\begin{tabular}{|c|c|c|}
\hline Modelos & $\begin{array}{c}\text { Energia } \\
(\mathrm{Kcal} / \mathrm{mol})\end{array}$ & Pareto \\
\hline 01 & 40,51 & 00 \\
\hline 02 & 39,81 & 00 \\
\hline 03 & 96,86 & 00 \\
\hline 04 & 36,36 & 00 \\
\hline 05 & 24,69 & 00 \\
\hline 06 & 20,48 & 00 \\
\hline 07 & 29,66 & 00 \\
\hline 08 & 60,01 & 00 \\
\hline 09 & 20,53 & 00 \\
\hline 10 & 29,35 & 00 \\
\hline
\end{tabular}

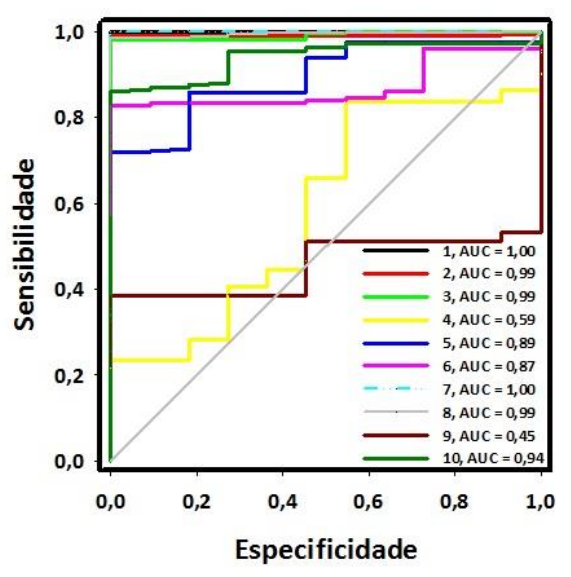

Figura 01: A- Características de cada modelo farmacofórico fornecidas pelo GALAHAD ${ }^{\mathrm{TM}}$. B- Curva ROC dos modelos farmacofóricos alinhados com um banco de moléculas contendo 11 ativos e 550 decoys. A linha diagonal representa um modelo que não seria melhor do que um ensaio randômico (AUC $<0,5)$.

Apesar dos modelos 01, 01, 03, 05, 06, 07, 08 e 10 serem preditivos (AUC > 0,7), a AUCé uma métrica contínua e global e, portanto não avalia o desempenho nas fases iniciais. Nesta perspectiva, apenas os modelos 01, 02, 03, 07 e 08 (BEDROC > 0,50) foram preditivos nas fases iniciais da triagem por modelo farmacofórico e, por conseguinte selecionados para a próxima etapa. 
Um modelo farmacofórico útil para a triagem virtual deve ser preditivo e capaz de contribuir para identificar moléculas potentes. Assim, apenas o modelo 02 apresentou melhores resultados (pIC50 x QFIT) tanto para AChE quanto para BuChE (Figura 2). A fim de caracterizar o melhor modelo farmacofórico, uma sobreposição do inibidor potente para a $\mathrm{AChE}$ e BuChE foi realizada $(\mathrm{Ki} \mathrm{AChE}=32,02 \mathrm{nM} ; \mathrm{BuChE}=24,7 \mathrm{nM})$.
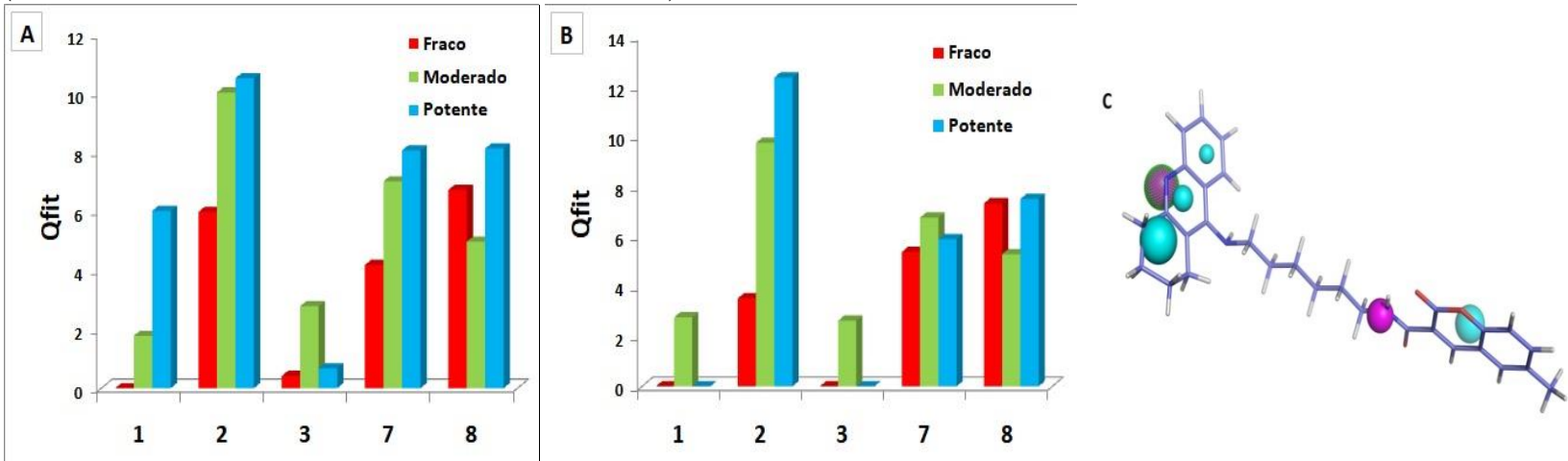

Figura 02:Gráficos representando o alinhamento dos modelos farmacofóricos remanescentes com inibidores de AChE (A) e BuChE (B). Sobreposição de um inibidor potente (roxo = carbono, branco = hidrogênio, vermelho = oxigênio, azul = nitrogênio; $\mathrm{Ki} \mathrm{AChE}=32,02 \mathrm{nM} ; \mathrm{BuChE}=24,7 \mathrm{nM}$ ) no modelo farmacofórico $02(\mathrm{C})$. Esferas em ciano representam centros hidrofóbicos (HY), esferas magenta representam doadores de ligação de hidrogênio (HBD), e pontos verdes representam aceptores de ligação de hidrogênio (HBA). O tamanho das esferas varia de acordo com o raio de tolerância calculado pelo GALAHAD.

Este modelo possui um aceitador de ligação de hidrogênio (HBA), dois doadores de ligação de hidrogênio (HBD) e quatro centros hidrofóbicos (HY). Dessa forma, as características de centros hidrofóbicos e aceitador de ligação de hidrogênio são requisitos essenciais para a inibição (ALMEIDA, 2015), enquanto a característica de doador de ligação de hidrogênio está relacionada a inibidores potentes.

Após a filtragem do banco da Sigma-Aldrich ${ }^{\circledR}$ (https://www.sigmaaldrich.com)pelo modelo farmacofórico dual,1881 obtiveramQFIT > $0(1,75<$ QFIT < 94,14), sendo assim selecionadas para a etapa de acoplamento molecular.A avaliação da sobreposição da melhor pose do ligante cristalográfico foi avaliada com o AutoDockVina 1.1.2 (TROTT \& OLSON, 2010), com um RMSD $=1,97 \AA$ para $\mathrm{AChE}$ e RMSD = 1,77 $\AA$ para BuChE. Em seguida, o mesmo conjunto teste da etapa de avaliação dos modelos farmacofóricos (ATIVOS+DECOYS)foi submetido ao acoplamento molecular e resultados avaliados através da construção da curva ROC e cálculo da AUC, obtendo os resultados de $\mathrm{AUC}=0,88$ para $\mathrm{AChE}$ e $\mathrm{AUC}=0,86$ para $\mathrm{BuChE}$.

Com base nos parâmetros avaliados, a pontuação dos 1881 compostos acoplados ao sitio ativo da $\mathrm{AChE}$ e $\mathrm{BuChE}$ variaram entre $-12,5$ < energia de afinidadeem $\mathrm{kcal} / \mathrm{mol}>-0,1$. Após aplicação da mediana ( $\mathrm{AChE}=-9,0$ e $\mathrm{BuChE}=-8,6)$, restaram 943 moléculas para AChE e 970 moléculas para BuChE. Tais moléculas foram analisadas quanto aeficiência de ligação para avaliar a contribuição dos átomos das moléculas para a ligação ao sítio ativo, onde foi possível selecionar 707 moléculas com características comuns tanto a AChE como a BuChE. Com base nadisponibilidade de aquisição e custo (< \$100) dessas moléculas, ZINC50499447 e ZINC71787304 foram adquiridas para ensaios subsequentes (ex. ensaios enzimáticos).

$\mathrm{Na}$ busca por aumentar as taxas de sucesso na priorização de moléculas bioativas e biodisponíveis, a utilização da Regra dos cinco de Lipinski e adicionalmente a complementaridade com os parâmetros de Veber et al., (2002) é capaz de predizer virtualmente a biodisponibilidade oral de moléculas de uma forma rápida e com baixo custo (LIPINSKI, 1997). Dessa forma, foram calculados os descritores físico-químicos para aplicação dos filtros moleculares sendo queas estruturas adquiridas demonstraram ser preditivas tanto para a inibição de $\mathrm{AChE} \mathrm{e} \mathrm{BuChE} \mathrm{quanto}$ para uma biodisponibilidade oral favorável. 

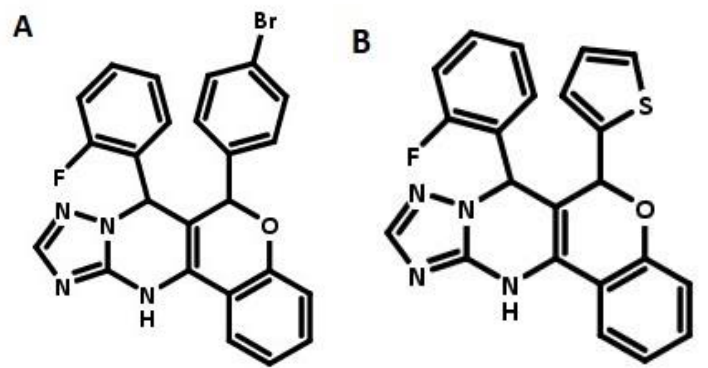

Figura 03: Moléculas adquiridas para testes enzimáticos. ZINC50499447 (A); ZINC71787304 (B).

A utilização do alinhamento molecular flexível Foi capaz de identificar os requisitos farmacofóricos necessários para a identificação de moléculas ativas frente a $\mathrm{AChE}$ e BuChE. As características apresentadas pelo modelo escolhido HY e HBA estão relacionadas ao reconhecimento pelo alvo, enquanto que a característica HBD predizem a potencia do inibidor, portanto, a presença destes requisitos é fundamental para oreconhecimento de moléculas duais e potentes frente a AChE e BuChE. A técnica de acoplamento molecular apresentou resultados satisfatórios, de modo que as moléculas testadas demonstraram afinidade pelo sítio alvo tanto de $\mathrm{AChE}$ quanto de BuChE.

\section{REFERÊNCIAS}

ALMEIDA, J. R. Planejamento, ensaio e otimização in silico de novos protótipos inibidores da enzima acetilcolinesterase. Tese (Doutorado em Ciências). Faculdade de Ciências Farmacêuticas de Ribeirão Preto, Ribeirão Preto, 2015.

CHEMAXON. Marvin Sketch version 15.4.20: ChemAxon, 2015.

FALCO, A. de et al. Doença de Alzheimer: hipóteses etiológicas e perspectivas de tratamento. Quim. Nova.v. 39.n. 1. p. 63-80, 2016.

HOPKINS, A. L. et al. The role of ligand efficiency metrics in drug discovery.Nature Reviews Drug Discovery.v. 13, p. 105-121. 2014.

LÄTTI, S. NIINIVEHMAS, S. PENTIKÄINEN, O. T. Rocker: Open source, easy-to-use tool for AUC and enrichment calculations and ROC visualization. Journal of Cheminformatics, v. 8, n. 45, 2016.

LIPINSKI, C. A., et al. Experimental and computational approaches to estimate solubility and permeability in drug discovery and development settings. Adv. Drug Deliv.Rev. v. 23. p. 3-26, 1997. MYSINGER, M. M. et al. DUDEnhanced - better ligands and decoys for better benchmarking. Journal of Medicinal Chemistry, v. 55, p. 6582-6594, 2012.

NAJAFI, Z. et al. Novel tacrine-1,2,3-triazole hybrids: In vitro, in vivo biological evaluation and docking study of cholinesterase inhibitors. European Journal of Medicinal Chemistry. v. 125. p. 1200-1212, 2017.

RODRIGUES, R.P. et al. Estratégias de Triagem Virtual no Planejamento de Fármacos. Revista Virtual de Química, v. 4, n. 6, p. 739-776, 2012.

SONDERGAARD, C. R. et al. Improved Treatment of Ligands and Coupling Effects in Empirical Calculation and Rationalization of pKa Values. Journal of Chemical Theory and Computation, v. 7 , n. 7, p. 2284-2295, 2011.

SUN, Q. et al. Syntheses of coumarin-tacrine hybrids as dual-site acetylcholinesterase inhibitors and their activity against butylcholinesterase, $A \beta$ aggregation, and $\beta$-secretase. Bioorganic \& Medicinal Chemistry, n. 22, p. 4784-4791, 2014.

TRIPOS. SYBYL-X 2.0, St Louis, MO, USA, p. 190,2010.

TROTT, O.; OLSON, A. J. Software news and update AutoDockVina: Improving the speed and accuracy of docking with a new scoring function, efficient optimization, and multithreading. Journal of computational chemistry, v.31, n.2, p.455-461, 2010.

VEBER et al. Molecular Properties That Influence the oral bioavailability of Drug Candidates. $J$. Med. Chem. v. 45. 2002. 